\title{
MEASUREMENT OF COSMIC SHEAR WITH THE SPACE TELESCOPE IMAGING SPECTROGRAPH
}

\author{
Jason Rhodes, ${ }^{1,2,3}$ Alexandre Refregier, ${ }^{3,4,5}$ Nicholas R. Collins, ${ }^{1,6}$ Jonathan P. Gardner, ${ }^{1}$ \\ Edward J. Groth, ${ }^{7}$ and Robert S. HiLl ${ }^{1,6}$ \\ Received 2003 August 1; accepted 2003 December 19
}

\begin{abstract}
Weak lensing by large-scale structure allows a direct measure of the dark matter distribution. We have used parallel images taken with the Space Telescope Imaging Spectrograph (STIS) on the Hubble Space Telescope to measure weak lensing, or cosmic shear. We measure the shapes of 26,036 galaxies in 1292 STIS fields and measure the shear variance at a scale of 0.51. The charge transfer efficiency (CTE) of STIS has degraded over time and introduces a spurious ellipticity into galaxy shapes during the readout process. We correct for this effect as a function of signal-to-noise ratio and CCD position. We further show that the detected cosmic shear signal is nearly constant in time over the approximately $4 \mathrm{yr}$ of observation. We detect cosmic shear at the $5.1 \sigma$ level, and our measurement of the shear variance is consistent with theoretical predictions in a $\Lambda$ CDM universe. This provides a measure of the normalization of the mass power spectrum $\sigma_{8}=(1.02 \pm 0.16)\left(0.3 / \Omega_{m}\right)^{0.46}(0.21 / \Gamma)^{0.18}$. The $1 \sigma$ error includes noise, cosmic variance, systematics, and the redshift uncertainty of the source galaxies. This is consistent with previous cosmic shear measurements, but tends to favor those with a high value of $\sigma_{8}$. It is also consistent with the recent determination of $\sigma_{8}$ from the Wilkinson Microwave Anisotropy Probe $(W M A P)$ experiment.
\end{abstract}

Subject headings: cosmology: observations — dark matter — gravitational lensing

On-line material: color figure

\section{INTRODUCTION}

Weak gravitational lensing by large-scale structure has become an important tool in understanding the amount and distribution of dark matter (see Mellier et al. 2003 and Refregier 2003 for reviews). Most cosmic shear measurements have been performed using wide-field, ground-based telescopes. However, the measurement of galaxy shapes from the ground is limited by atmospheric seeing, while the Hubble Space Telescope (HST) has a much smaller point-spread function (PSF; $<0$ " 1 , as opposed to $\sim 0$ "'8 for ground-based telescopes). Several groups have thus used HST images to study cosmic shear (Rhodes, Refregier, \& Groth 2000, 2001, hereafter RRG00 and RRG01, respectively; Refregier, Rhodes, \& Groth 2002, hereafter RRG02; Hämmerle et al. 2002). Recent measurements of cosmic shear from the ground (Bacon et al. 2003; Hoekstra et al. 2002; van Waerbeke et al. 2002; Jarvis et al. 2003; Brown et al. 2003) and from space (RRG02) have been used to derive an estimate of the normalization $\sigma_{8}$ of the matter power spectrum with an uncertainty comparable to that of more traditional methods, such as the measurement of cluster abundances (see RRG02 for a discussion).

In this paper, we present the results of a weak-lensing analysis of images taken with the Space Telescope Imaging Spectrograph (STIS) on HST. The STIS data we use have been described by Pirzkal et al. (2001). Hämmerle et al. (2002)

\footnotetext{
${ }^{1}$ Laboratory for Astronomy and Solar Physics, Code 681, NASA Goddard Space Flight Center, Greenbelt, MD 20771; rhodes@astro.caltech.edu.

${ }^{2}$ NASA/National Research Council Research Associate.

${ }^{3}$ California Institute of Technology, 1201 East California Boulevard, Pasadena, CA 91125.

${ }^{4}$ Service d'Astrophysique, CEA Saclay, 91191 Gif-sur-Yvette, France.

5 Institute of Astronomy, Madingley Road, Cambridge CB3 OHA, UK.

${ }^{6}$ Science Systems and Applications, Incorporated.

7 Department of Physics, Princeton University, Jadwin Hall, P.O. Box 708, Princeton, NJ 08544.
}

have presented an analysis of a subset (121 of 1292 fields) of these data, reporting a $4 \% \mathrm{rms}$ shear at the $1.5 \sigma$ level. Because there are a number of different methods for measuring shear and correcting for systematic effects, it is beneficial to compare the results we present here with the results of other groups examining data using the same instrument.

This paper is organized as follows. In $\S 2$, we describe the STIS data used in this study. We detail the STIS parallel archive maintained by the STIS Investigation Definition Team (IDT) at Goddard Space Flight Center, including the methods used to reduce and catalog STIS images. Section 3 describes the procedure used to measure the weak-lensing shear in the STIS data, including the method for removal of systematic effects. In $\S 4.1$, we present the results, including constraints on cosmological parameters. We draw our conclusions and briefly comment on the future of weak-lensing measurements in $\S 5$.

\section{DATA}

The STIS images used in this study were taken primarily in parallel mode, meaning that another instrument on HST was the prime observing instrument. Thus, the images are essentially randomly positioned on the sky, separated from the primary observation by $5^{\prime}-8^{\prime}$, depending on the primary instrument. This is ideal for a study of cosmic shear, because many random pointings on the sky minimize the error due to cosmic variance.

There are four stages to the data reduction: (1) input image co-alignment for a particular field; (2) preliminary image reduction that involves bias and dark subtraction, hot-pixel correction, flat-field division, and correction for geometric distortion; (3) cosmic-ray (CR) removal and image combination; and (4) object detection.

Step 1 involves determining the relative offsets using sources identified in dithered images. Copies of the input 
images are precleaned to minimize the effect of CRs on the source identification step. Small clusters of pixels with high data values relative to surrounding pixels are first identified and then corrected using interpolation. This precleaning may modify or remove real sources, but these image copies are used only for the purpose of co-alignment. Source Extractor (SExtractor; Bertin \& Arnouts 1996) is used to identify sources in the precleaned image copies. Those sources in common on all images are used to find individual image offsets. The average offset is subtracted from the individual offsets, so that all images will be shifted to a common field center.

For step 2, we used the Interactive Data Language (IDL) version of CALSTIS (Lindler 2003), ${ }^{8}$ which was developed by the STIS IDT. A one-dimensional fit is made to the CCD overscan region and subtracted from each column of the image array to account for temporal variations between the observation and the two-dimensional bias image. A twodimensional bias frame is then subtracted from the science image. Next, the dark image subtraction is performed, and hot pixels are corrected. The hot-pixel tables from the Space Telescope Science Institute archive list new hot pixels created by the constant on-orbit CR flux since the last dark image was obtained. For a given observation, the hot pixels corrected are those in common between the two lists made closest in time before and after the observation. Hot pixels with a dark rate greater than 0.02 counts $\mathrm{s}^{-1}$ were corrected by linear interpolation among the eight adjacent pixels. The resultant image is divided by two flat-field images: one that accounts for pixelto-pixel variations and another that corrects the low-frequency spatial variations across the field of view due to vignetting. A final hot-pixel correction is performed to preclean the data before CR rejection. Using a separate IDL program, small clusters of pixels ( $\leq 3$ pixels wide) with high data values relative to surrounding pixels are first identified and then corrected using interpolation. This procedure is especially useful in removing hot or bad pixels that would not otherwise be eliminated in the $\mathrm{CR}$ rejection step when there is no dither. This is the same algorithm described in step 1 for removing CRs before running SExtractor, but in this case, the threshold for correction is set to a higher value so that valid source pixels are not modified. The correction for detector/opticsinduced shear (or geometric distortion) is performed with bilinear interpolation using the values given by Malumuth \& Bowers (1997). The distortion coefficients are derived using the astrometric shifts of stellar images. All images are then shifted to a common field center using bilinear interpolation for the subpixel offsets derived in step 1 .

Once the individual images have been co-aligned, they are combined with the IDL routine cr_reject.pro (step 3). This program emulates the STSDAS task stsdas.hst_calib.wfpc.crrej and is equipped to handle input images with different exposure times. The main difference between the two algorithms is in the initial step of sky background determination. The procedure stsdas.hst_calib.wfpc.crrej uses the modal value of all of the image pixels, while cr_reject.pro uses an estimate of modal value of a subset of image pixels. Using a subset of image pixels reduces the bias in the pixel distribution caused by foreground source flux. The estimate uses an algorithm from DAOPHOT I (Stetson 1987). The average and standard deviation of the

\footnotetext{
${ }^{8}$ Lindler (2003) is available at http://hires.gsfc.nasa.gov/stis/software/ doc_manuals.html.
}

pixel value distribution are computed, and outliers are removed using an iterative sigma-clipping method. If the distribution is Gaussian (uncontaminated by foreground sources), the mean, median, and mode should be the same. If, after 20 iterations, the mean and median are the same, this value is taken as the sky for the image. If the mean of the distribution is larger than the median (a non-Gaussian distribution), the true sky is estimated as 3 times median -2 times mean. The scalar background so derived is subtracted from each input image. Pixels are rejected as CRs if their value is greater than an input number of $\sigma$ from a reference value. The constant read noise, the statistical noise (square root of the counts), and a noise proportional to the counts comprise the $\sigma$. This last noise component accounts for differing PSFs or subpixel image shifts. It is expressed by the mult_noise variable in cr_reject.pro, which we set to 0.03 . If there are seven or more input images, the first guess at a CR-free or reference image is the pixel-by-pixel median of all the input images; otherwise, the minimum is used. The first pixel rejection pass cuts all pixels greater than $8 \sigma$ from this reference image. A second pass uses the mean of the cleaned images as the reference image and a rejection criterion of $6 \sigma$. The third and final pass uses a $4 \sigma$ rejection criterion on the mean of the cleaned images from the second pass. The sky values that were subtracted from each input image before $\mathrm{CR}$ rejection are added back into the final set of cleaned images. The final image combination is the weighted average of the cleaned images. The weight parameters are the sky noise and the read noise. The final image pixels are in units of counts per second.

For step 4 we use SExtractor (Bertin \& Arnouts 1996) for object detection, photometry and star/galaxy separation and to compute positions for each detected object. SExtractor subtracts a smoothed, two-dimensional background with a mesh size of $1^{\prime \prime}$ (back_size = 20) and filter size setting of back_filter size $=3$. The image is then convolved with a 1 .'25 251 1".25 0.5 FWHM Gaussian filter. The detection and analysis thresholds are both set to $0.7 \sigma$ above the measured sky noise. We require that five contiguous pixels meet this threshold criterion to be considered a valid source. In order to separate blended sources, we set the number of deblending subthresholds to 32 and the minimum contrast parameter for deblending to 0.005 . The input stellar seeing disk FWHM, used for star-galaxy separation, is 0.07 . The photometry reported is taken from the mag_auto column of the output catalog; mag_auto is the source magnitude measured inside a unique elliptical aperture for every object (Kron 1980). The twoelement input parameter phot_autoparams controls the elliptical apertures. The first element is the $k$-factor described in Bertin \& Arnouts (1996), and the second is the minimum possible radius (in pixels) for an elliptical, or Kron, aperture. We use the default values of 2.5 and 3.5, respectively, for the two elements. A 12 pixel annulus outside the elliptical aperture is used to determine the local sky background around each object.

We examined 2335 fields, imaged in 21 HST programs (7781, 7782, 7783, 7908, 7910, 7911, 8062, 8064, 8084, 8091, $8393,8406,8470,8545,8549,8562,8796,8808,8870,8884$, and 9248). Several of these programs are described elsewhere: the STIS Parallel Survey (SPS; Gardner et al. 1998; Teplitz et al. 2003a, 2003b) obtained filterless imaging and slitless spectra of random fields on the sky, and program 8562 (PI Schneider; continued as 9248) was conducted to gather data specifically to search for cosmic shear (Pirzkal et al. 2001). Of these fields, 1494 pass a visual inspection that indicates that 
they are not contaminated by stars or large objects. In our measurement of cosmic shear, we use 1292 of these fields that pass several other cuts described below.

The selected fields were imaged between 1997 August 9 and 2001 May 15. We do not use more recent data because of possible changes in systematic effects that may have occurred when STIS was forced to go to backup electronics as a result of a malfunction in 2001 July (Brown 2001). ${ }^{9}$ Each field has between 2 and 86 individual exposures, with total exposure times ranging from 265 to $34,200 \mathrm{~s}$. The distribution of exposure times and number of exposures are shown in Figure 1.

Imaging is done using the 50CCD clear mode, a very wide bandpass sensitive to light in the wavelength range $220 \mathrm{~nm}<$ $\lambda<1100 \mathrm{~nm}$ and limited by the sensitivity of the CCD (Baum et al. 1996). We co-add exposures that are within $5^{\prime \prime}$ of each other. Only exposures taken within 6 months of each other are co-added to produce the final image. This limit is chosen to avoid co-adding exposures with large differences in their calibration files.

Using the results of RRG02 and the fact that $\langle M\rangle \approx\langle I\rangle+1$ for typical galaxy colors, where $M$ and $I$ are the STIS and WFPC2 magnitudes, respectively, we find that the median magnitude $M_{m}$ of the galaxies in each STIS field is related to their median redshift $z_{m}$ by

$$
z_{m} \approx 0.72+0.15\left(M_{m}-23.0\right)
$$

We show a histogram of the median redshifts of the selected fields in Figure 2.

${ }^{9}$ Brown (2001) is available at http://www.stsci.edu/hst/stis/documents/isrs.
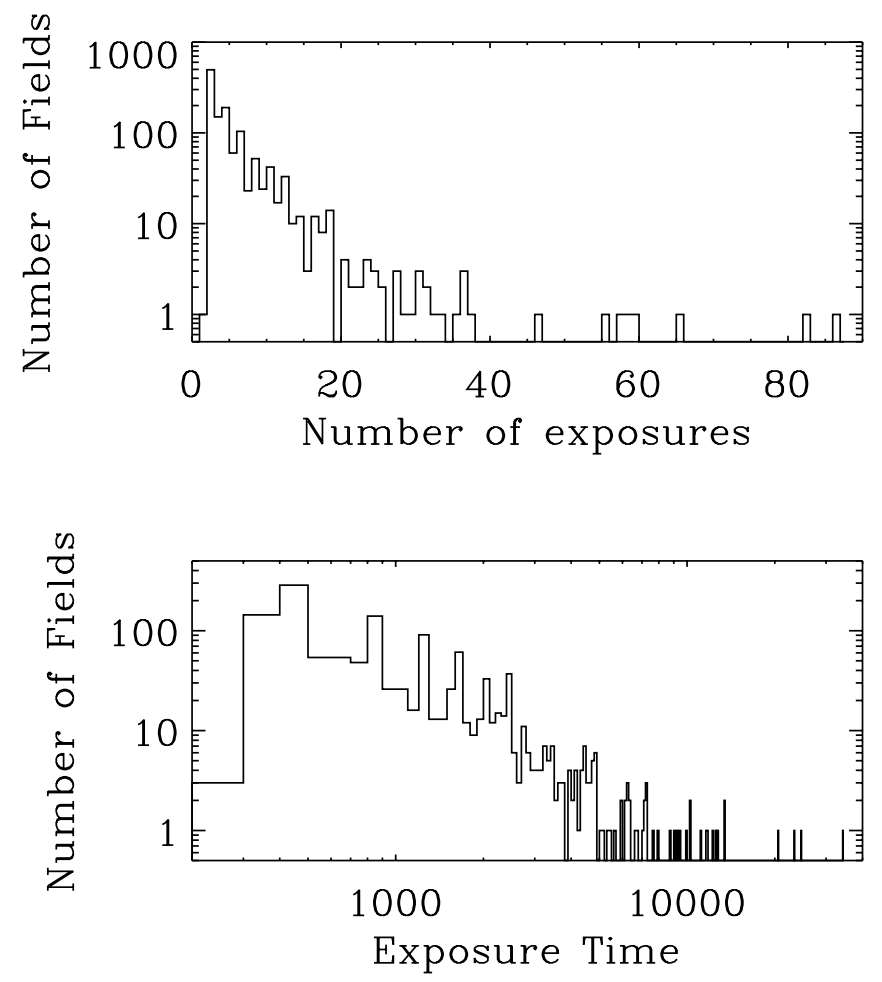

FIG. 1.-Top: Number of individual exposures going into each image. Bottom: Exposure time for each individual image.

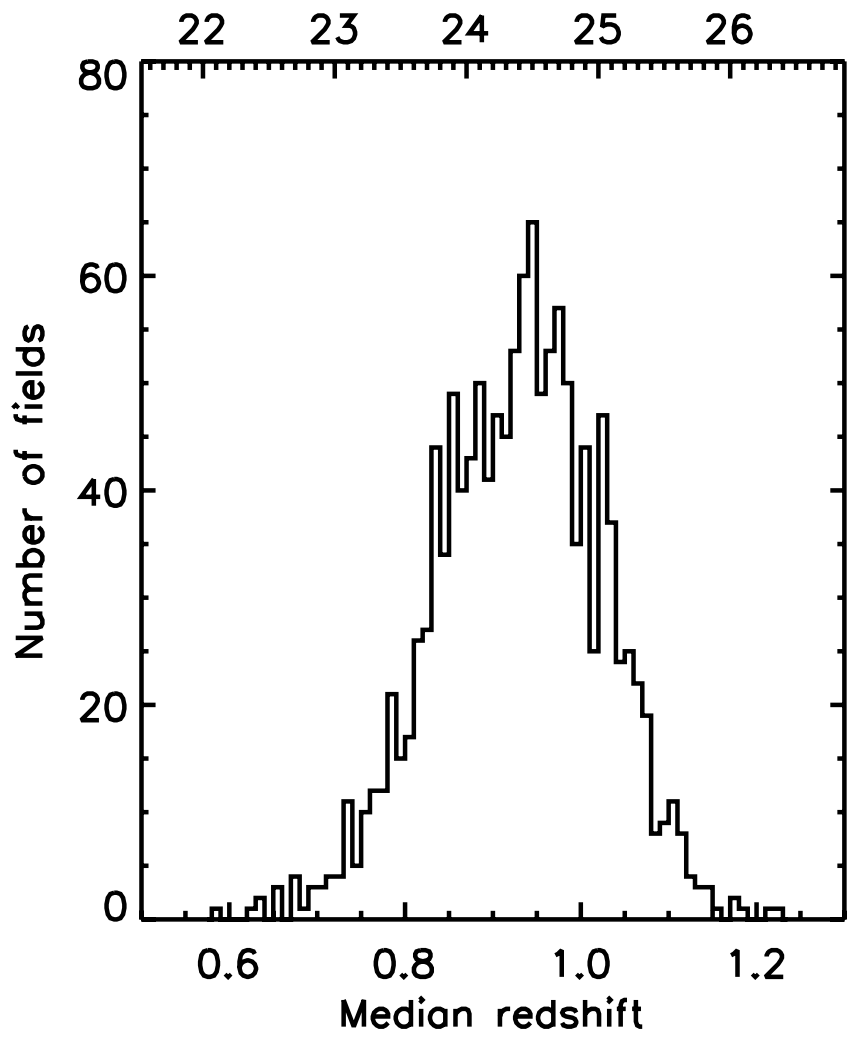

FIG. 2.-Distribution of the median redshifts of the 1292 fields we use for cosmic shear measurements. Redshift $z_{m}$ is estimated from the median magnitude of the selected galaxies in the field via eq. (1).

We visually examined 2335 fields in the STIS archive. Our selection criteria were (1) two or more exposures, (2) unbinned pixels (to maintain the full STIS resolution), and (3) imaging performed with the clear 50CCD filter. We discarded fields that have a very bright saturated star, fields that are crowded by stars (common for fields with galactic latitude $|b|<20^{\circ}$ ), and fields with a large object that covers a substantial portion of the field. This left us with 1494 fields. Because of repeat visits by the HST separated by more than 6 months and visits to fields that are separated by only of the order of tens of arcseconds, some of these fields overlap. So as not to doublecount fields, we discarded one of any pair of fields that lie closer than $1^{\prime}$ to each other (the STIS field of view is $50^{\prime \prime} \times$ $\left.50^{\prime \prime}\right)$. When considering which field to discard, we kept the field with the greater total exposure time.

For several reasons, including thermal effects, the HST undergoes jitter, or shaking (Nelan \& Makidon 2002). This jitter tends to blur images and introduces changes into measured object shapes. The median rms jitter in one direction is 3-4 mas, but the rms jitter value can be greater than 100 mas. We thus discard fields in which any of the input exposures had an rms jitter greater than 10 mas (0.2 STIS pixels) in either of two orthogonal directions or for which no jitter information is available. After these cuts, we were left with 1292 fields.

\section{PROCEDURE}

\subsection{Shape Measurement}

We follow the method described in RRG00 to measure the object shapes and sizes and to correct for systematic effects. We measure the second- $\left(I_{k l}\right)$ and fourth- $\left(I_{k l m n}\right)$ order moments 
for each object. These moments are defined as a sum over pixels $p$ given by, for example,

$$
I_{k l}=\frac{\sum_{p} x_{k} x_{l} I(\boldsymbol{x}) w(\boldsymbol{x})}{\sum_{p} I(\boldsymbol{x}) w(\boldsymbol{x})},
$$

where $I(\boldsymbol{x})$ is the intensity in a pixel with position $\boldsymbol{x}=$ $\left(x_{1}, x_{2}\right)=(x, y)$ with respect to the centroid of the object and $w(\boldsymbol{x})$ is a Gaussian weighting function. Similar equations hold for the remaining moments. The standard deviation of the weight function is chosen to be (in pixels)

$$
\sigma_{w}=\max (3, \sqrt{A / \pi})
$$

where the area $A$ is determined from the SExtractormeasured semimajor $(a)$ and semiminor $(b)$ axes as $A=$ $\pi[(a+b) / 2]^{2}$. The minimum weight function size of 3 pixels $(0,15)$ is the optimal weight function we found for point sources (stellar images). This is similar to the minimum weight function size of 2 pixels $(0.2)$ found in RRG00 for WFPC 2.

These measured moments are used to compute the twocomponent ellipticity of each galaxy, given by

$$
e_{1}=\frac{I_{11}-I_{22}}{I_{11}+I_{22}}, \quad e_{2}=\frac{2 I_{12}}{I_{11}+I_{22}}
$$

The first component of this ellipticity $\left(e_{1}\right)$ indicates elongation along the $x$ - (positive $e_{1}$ ) and $y$ - (negative $e_{1}$ ) axes; $e_{2}$ indicates elongation along axes $45^{\circ}$ and $-45^{\circ}$ from the $x$-axis. We also define the rms size $d$ of the object as

$$
d^{2}=\frac{1}{2}\left(I_{11}+I_{22}\right) \text {. }
$$

There are several systematic effects that affect the measured shapes of galaxies. The PSF of the telescope introduces smearing that can be deconvolved into an isotropic and an anisotropic component. The telescope's optics introduce an instrumental shear into the galaxies. Our method to correct for these effects is presented in RRG00. Here we describe the particular steps taken for the STIS data set.

\subsection{PSF Model}

Ground-based surveys typically use stars in the survey fields to measure and correct for the PSF. Because of the small field of view of HST, space-based surveys do not have enough stars per field to do such a correction and have relied on separate observations of high signal-to-noise ratio $(\mathrm{S} / \mathrm{N})$ stellar fields to measure the PSF (see, e.g., RRG00; RRG02; Hämmerle et al. 2002). However, in examining the STIS PSF, we found that the PSF shape depends on the $\mathrm{S} / \mathrm{N}$. As shown in Figure 3 , the average ellipticity $\left(e_{1}\right)$ of stars in the survey fields changes from negative (elongation along the $y$-axis) to positive (elongation along the $x$-axis) as $\mathrm{S} / \mathrm{N}$ increases. This result is consistent with a previously known charge transfer efficiency (CTE) effect in STIS (Kimble, Goudfrooij, \& Gilliland 2000). Objects with few counts (because of short exposure times or low $\mathrm{S} / \mathrm{N}$ ) bleed in the $y$-direction, causing an elongation of the object in that direction. This CTE effect has been shown to be worse in the $(y<512)$ half (hereafter the bottom half) of the STIS CCD (B. Woodgate 2002, private

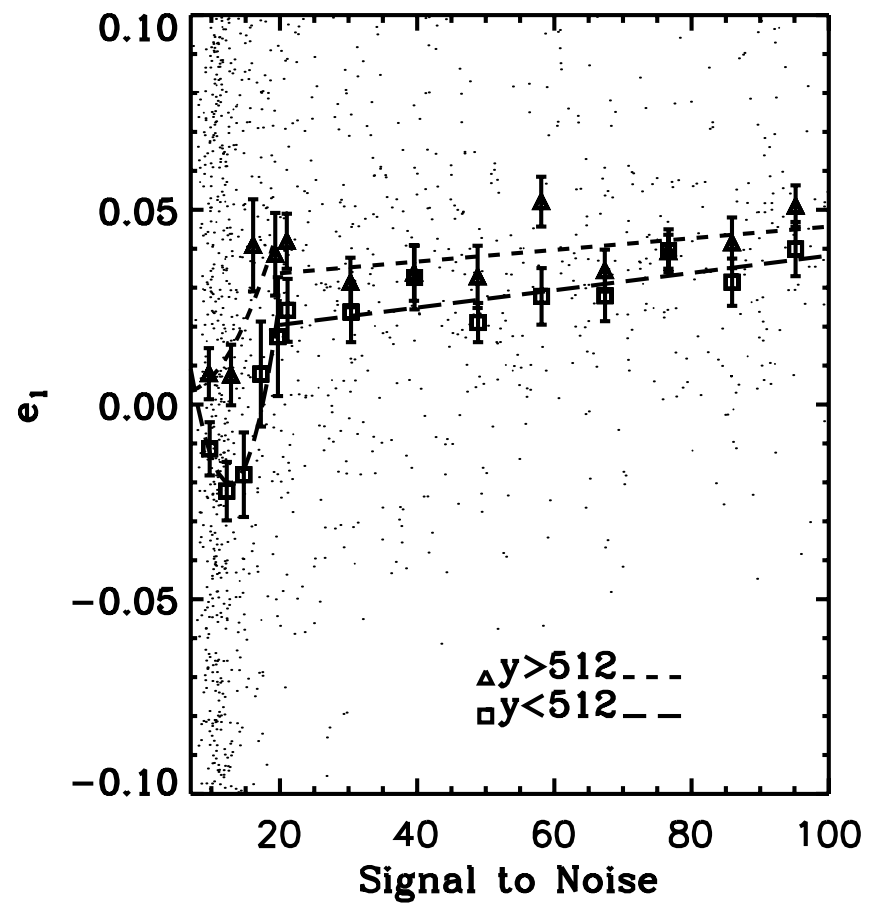

FIG. 3.- Stellar ellipticity $e_{1}$ as a function of $\mathrm{S} / \mathrm{N}$ for stars in galaxydominated fields. The triangles represent binned averages in the top half of the chip and squares those in the bottom half of the chip. The error bars represent $1 \sigma$ errors in the mean. The dashed lines represent linear fits for $20<\mathrm{S} / \mathrm{N}<$ 100 and quadratic fits for $\mathrm{S} / \mathrm{N}<20$. These fits are used to derive corrections for the second-order moments $I_{k l}$ based on $\mathrm{S} / \mathrm{N}$ and $y$-position.

communication). Figure 3 confirms that the elongation in the $y$-direction is indeed more pronounced in the bottom half of the STIS CCD, providing further evidence that the CTE is indeed the source of the $\mathrm{S} / \mathrm{N}$ dependence of the PSF.

The CTE effect described above complicates the modeling of the PSF, and thus the correction of galaxy shapes for PSF smearing, for two reasons. The first is that we cannot simply measure high-S/N stellar PSFs and use these to correct low$\mathrm{S} / \mathrm{N}$ galaxies. The second is that we cannot use separate stellar fields to measure the PSF. Stars in star-dominated fields (such as images of globular clusters) do not suffer the same CTE effects as stars in sparsely populated fields, even at low $\mathrm{S} / \mathrm{N}$. This is because the higher background levels in stellar fields fill in the charge traps, which cause the CTE effect. Thus, low-S/N stars in high-background fields have a PSF similar to the PSF of high-S/N stars regardless of background. Since the bulk of our galaxies are low-S/N galaxies in low-background fields, that is the regime in which we must model the PSF.

For $\mathrm{S} / \mathrm{N}>100$, we see no evidence for an $e_{1}$ dependence on either $\mathrm{S} / \mathrm{N}$ or $y$-position. Therefore, we consider the high$\mathrm{S} / \mathrm{N}$ PSF to be the "true" STIS PSF and model the $\mathrm{S} / \mathrm{N}$ and $y$ dependence of the PSF as perturbations about this true PSF. We select 534 high- $(>100) \mathrm{S} / \mathrm{N}$ stars from our survey and fit the eight stellar moments from these stars to fifth-order polynomials in the $x$ - and $y$-positions, as described in RRG00. Since we are attempting only a first-order correction for the effects of $\mathrm{S} / \mathrm{N}$ and $y$-position, we do not correct the fourthorder moments for these effects. The low-S/N moment at any position $(x, y)$ is given by the high-S/N moment at that position, as calculated from the fifth-order polynomial fits, minus some correction factor. The correction factor is the difference between the average value of the moment at high $\mathrm{S} / \mathrm{N}$ and the 
TABLE 1

PSF Model Parameters for Equations (4) and (5)

\begin{tabular}{|c|c|c|c|}
\hline Parameter & S/N Range & $y$ Range & Fit \\
\hline$d^{2}$. & $10-100$ & All & $1.47+0.00072(\mathrm{~S} / \mathrm{N})$ \\
\hline \multirow[t]{4}{*}{ 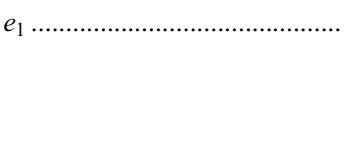 } & $8-20$ & $<512$ & $0.124-0.023(\mathrm{~S} / \mathrm{N})+0.00089(\mathrm{~S} / \mathrm{N})^{2}$ \\
\hline & & $>512$ & $0.010-0.0025(\mathrm{~S} / \mathrm{N})+0.00021(\mathrm{~S} / \mathrm{N})^{2}$ \\
\hline & $20-100$ & $<512$ & $0.016+0.0022(\mathrm{~S} / \mathrm{N})$ \\
\hline & & $>512$ & $0.031+0.00015(\mathrm{~S} / \mathrm{N})$ \\
\hline 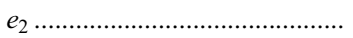 & All & All & 0 \\
\hline
\end{tabular}

average value of the moment at low $\mathrm{S} / \mathrm{N}$ at the $y$-position of the star on the chip. Thus,

$$
I_{k k}^{\text {low }}(x, y)=I_{k k}^{\mathrm{high}}(x, y)-\left[\bar{I}_{k k}^{\mathrm{high}}-\bar{I}_{k k}^{\mathrm{low}}(y, \mathrm{~S} / \mathrm{N})\right],
$$

for each diagonal component $k=1,2$.

We then write the moments as

$$
I_{11}=\left(1+e_{1}\right) d^{2}, \quad I_{22}=\left(1-e_{1}\right) d^{2},
$$

where the size $d$ is defined as in equation (5). This size is slightly dependent on $\mathrm{S} / \mathrm{N}$ but has no dependence on $y$-position. The size $d^{2}$ as a function of $\mathrm{S} / \mathrm{N}$ is well fitted by a linear equation, given in Table 1 . We fit $e_{1}$ to the $\mathrm{S} / \mathrm{N}$ in two different bins of $y$-position, $y<512$ and $y>512$. We use linear fits for the range $20<\mathrm{S} / \mathrm{N}<100$ and quadratic fits for the range $8<\mathrm{S} / \mathrm{N}<20$. Table 1 gives the best-fit functions, which are plotted on Figure 3. To calculate the average value of $e_{1}$ at a particular $\mathrm{S} / \mathrm{N}$ and $y$, we perform a linear interpolation or extrapolation between the two $y$ fits at that $\mathrm{S} / \mathrm{N}$ value, assuming that the $y<512$ fit is valid for $y=256$ and the $y>512$ fit is valid for $y=768$. We find that there is no $\mathrm{S} / \mathrm{N}$ or $y$ dependence for $e_{2}$, which is consistent with zero. Thus, no $I_{12}$ correction is necessary. These fits allow us to calculate the corrections to the high-S/N stellar moments using equation (6).

The average stellar ellipticity we measure at high $\mathrm{S} / \mathrm{N}$ $(\mathrm{S} / \mathrm{N}>100), 3 \%-5 \%$, is slightly higher than the $1 \%-2 \%$ measured by Hämmerle et al. (2002). We compare our measurement with theirs by obtaining one of the high-S/N stellar fields (o48b41010_3_ass) in their publicly available catalog and performing our analysis on that image. This image is drizzled to increase resolution and thus has a linear pixel size of 0 ". 025 (half of the intrinsic STIS pixel size). If we analyze this image using the method outlined above with a Gaussian weight function width $\sigma=6$ pixels (twice what we use in our nondrizzled images), we find an average stellar ellipticity $e_{1}=4 \%-5 \%$. This is stable for larger weight function widths, but if we use a smaller weight function width $(\sigma=3$ drizzled pixels) we measure an average $e_{1}=1.5 \%$. Thus, we are able to recover the value measured by Hämmerle et al. (2002) by using a sufficiently compact weight function. The weight function we use to measure stars represents a trade-off between the reduction of noise in moment measurements and the measurement of the full PSF anisotropy. As in RRG00, stellar moments are relatively stable for small perturbations in this weight function width.

\subsection{Correction for the PSF}

Using the stellar moments - corrected for weighting and detector shear as described in RRG00 and fitted to S/N and $y$-position as described above-we can correct the galaxy moments for the effects of the PSF. The first step is a correction for the anisotropic smearing of the PSF, and the second step is a correction for the isotropic portion of the PSF, which increases the galaxy size slightly. This correction method was specifically tuned to $H S T$ images with small PSFs and has produced excellent results in the correction of WFPC2 images for PSF effects (RRG01; RRG02). The corrected galaxy moments are used to calculate the ellipticity of each galaxy, which allows the calculation of the overall shear in each field, as described below.

\subsection{Shear Measurement}

We detected 63,895 objects in the 1292 fields we used. We discard stars and objects for which no jitter information is available or for which the jitter is very high. We further discard objects that have spurious ellipticities (nonphysical postcorrection ellipticities, usually caused by poor background subtraction), leaving us with 49,692 objects. We then discard objects that are too small to allow a shape measurement, i.e., with $d<1.7$ pixels (after corrections), leaving 35,033 objects. In RRG01 and RRG02, a magnitude cut was made to discard galaxies too faint to allow for shape measurement. Here, we opt to use an $\mathrm{S} / \mathrm{N}$ cut based on SExtractor values of flux and flux error. A cutoff value of $\mathrm{S} / \mathrm{N}=10$ left us with 26,036 galaxies.

We thus obtain the corrected ellipticity $e_{i}$ of each selected galaxy that passed all the cuts mentioned above. We then calculate the shear estimator $\gamma_{i}$ for each galaxy, using the equation

$$
\gamma_{i}=\frac{e_{i}}{\langle G\rangle},
$$

where the shear susceptibility factor $\langle G\rangle \simeq 1.4$ is calculated according to equation (28) of RRG00 and averaged over all galaxies in our final sample.

\section{RESULTS}

\subsection{Shear Measurement and Cosmological Parameter Estimation}

To quantify the cosmic shear statistics, we follow the procedure of RRG02. The cosmic shear variance $\sigma_{\text {lens }}^{2}$ is obtained from a weighted sum of the estimators $\sigma_{\text {lens } f}^{2}$ for each field $f$ as

$$
\sigma_{\text {lens }}^{2}=\sum_{f} w_{f} \sigma_{\text {lens }, f}^{2}
$$

where the weights $w_{f} \propto \sigma_{\text {noise }, f}^{-4}$ are normalized so that $\sum_{f} w_{f} \equiv 1$. We define the noise variance $\sigma_{\text {noise }, f}^{2} \equiv\left\langle\left|\gamma_{f}^{\text {noise }}\right|^{2}\right\rangle$, which is measured from the data by computing the error in the 
mean $\gamma_{i f}$ from the distribution of galaxy shears in each field (RRG02). This provides a nearly optimal weighting scheme that allows us to use all fields that are not contaminated by stars or bright objects. Fields with few objects that pass our selection criteria are appropriately down-weighted.

With our STIS data set, we find

$$
\sigma_{\text {lens }}^{2}=(5.43 \pm 1.06 \pm 1.74) \times 10^{-4},
$$

where the first error corresponds to noise only, while the second includes noise, cosmic variance, and systematics. Our detection of cosmic shear (first error) is thus significant at the $5.1 \sigma$ level. The systematics error is dominated by the uncertainty in our PSF correction (see $\S 3$ ). To estimate it, we first noted that the uncertainty in our measurement of the PSF ellipticity $e_{*}$ in Figure 3 is $\Delta e_{*} \simeq 0.01$. The resulting uncertainty in the galaxy shears is $\Delta \gamma \simeq G^{-1}\left(\left\langle d_{*}\right\rangle /\langle d\rangle\right)^{2} \Delta e_{*} \simeq$ $1.0 \times 10^{-3}$ (see eq. [58] of RRG00), where the average rms size (see eq. [5]) of the PSF (with $\mathrm{S} / \mathrm{N}=10$; see Table 1) and of galaxies (after our cuts) is $\left\langle d_{*}\right\rangle \simeq 1.2$ pixels and $\langle d\rangle \simeq 3.2$ pixels, respectively.

Our measurement of the shear variance is shown in Figure 4. Recent measurements from other surveys with similar galaxy redshifts $\left(z_{m} \simeq 0.8-1\right.$; RRG02; van Waerbeke et al. 2002; Brown et al. 2003; Bacon et al. 2003) are also shown for comparison. The solid lines show the predictions for a $\Lambda \mathrm{CDM}$ (cold dark matter) model with $\Omega_{m}=0.3, \sigma_{8}=1, \Gamma=0.21$, and the above range of galaxy median redshift $z_{m}$. Our variance measurement is consistent with these other measurements and with the $\Lambda$ CDM prediction. It also agrees with the measurement of Hämmerle et al. (2002), who found $\sigma_{\text {lens }}^{2} \simeq$ $(15 \pm 12) \times 10^{-4}$ (from their Fig. 15). Note that their error bar

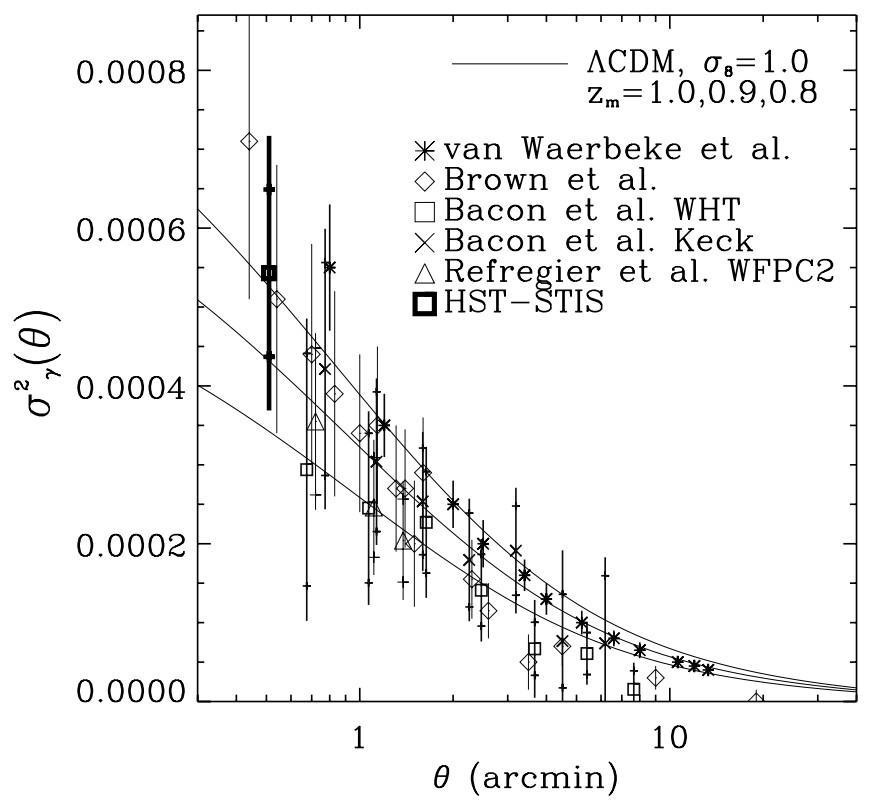

FIG. 4.-Cosmic shear variance $\sigma_{\text {lens }}^{2}$ as a function of the radius $\theta$ of a circular aperture. Our observed value (HST STIS) is shown, as well as that from other recent measurements: van Waerbeke et al. (2002), Bacon et al. (2003; WHT and Keck), Brown et al. (2003), and RRG02 (HST WFPC2). Also displayed are the predictions for a $\Lambda$ CDM model with $\Omega_{m}=0.3$, $\Gamma=0.21, \sigma_{8}=0.7$ and 1.0 , and a range of galaxy median redshifts $z_{m}=0.8$, 0.9 , and 1.0, using the Smith et al. (2003) nonlinear fitting function. When relevant, the inner error bars include statistical errors only, while the outer error bars include statistical errors and cosmic variance. [See the electronic edition of the Journal for a color version of this figure.] is considerably larger than ours, because their measurement is based on about $1 / 10$ of the fields we have used.

Our measurement allows us to set constraints on $\sigma_{8}$, the amplitude of matter fluctuations on $8 h^{-1} \mathrm{Mpc}$ scales. In a $\Lambda$ CDM model with $\Omega_{m}=0.3$ and $\Gamma=0.21$, the shear variance is given by

$$
\begin{aligned}
\sigma_{\text {lens }}^{2} \simeq & 5.17 \times 10^{-4}\left(\frac{\sigma_{8}}{1.0}\right)^{2.42}\left(\frac{\Omega_{m}}{0.3}\right)^{1.10} \\
& \times\left(\frac{\Gamma}{0.21}\right)^{0.44}\left(\frac{z_{m}}{1.0}\right)^{1.85},
\end{aligned}
$$

where $z_{m}$ is the median redshift of the galaxies, whose redshift distribution was assumed to be $p(z) \propto z^{2} e^{-\left(z / z_{0}\right)^{2}}$, with $z_{0}=z_{m} / 1.09$. The scale 0.51 is the effective radius of a circular cell corresponding to the chip size $\left(50^{\prime \prime}\right.$ on a side). The details of this calculation can be found in Bacon, Refregier, \& Ellis (2000). Note, however, that unlike these authors, we have used the more accurate fitting function of Smith et al. (2003) to compute the nonlinear corrections to the power spectrum.

For our sample and weighting scheme, the effective median magnitude of the galaxies is $M_{m} \simeq 24.8$. According to equation (1), this corresponds to a median redshift of $z_{m}=$ $1.0 \pm 0.1$, where the $1 \sigma$ error arises from the uncertainty in this equation. Our measurement of $\sigma_{\text {lens }}^{2}$ thus yields

$$
\sigma_{8}=(1.02 \pm 0.16)\left(\frac{0.3}{\Omega_{m}}\right)^{0.46}\left(\frac{0.21}{\Gamma}\right)^{0.18}
$$

where the error includes noise, cosmic variance, systematics, and the redshift uncertainty. This result is consistent at the $1 \sigma$ level with some earlier cosmic shear surveys (Bacon et al. 2003; RRG02; van Waerbeke et al. 2002), which derived values of $\sigma_{8}$ in the range $0.9-1.0$ for $\Omega_{m}=0.3$ (see Fig. 9 and Table 1 in Refregier 2003 for a summary of the latest cosmic shear results). It is also consistent, but only at the less than $2 \sigma$ level, with other cosmic shear surveys that found $\sigma_{8}$ in the range $0.7-0.8$ for the same value of $\Omega_{m}$ (Brown et al. 2003; Hamana et al. 2003; Jarvis et al. 2003; Hoekstra et al. 2002). The recent Wilkinson Microwave Anisotropy Probe (WMAP) results (Spergel et al. 2003) yield $\sigma_{8}=(0.91 \pm 0.21)\left(0.3 / \Omega_{m}\right)^{0.6}$, when taken alone (for their single-power-law model), and $\sigma_{8}=0.78_{-0.10}^{+0.08}\left(0.3 / \Omega_{m}\right)^{0.6}$, when combined with other cosmic microwave background (CMB) and large-scale structure data (for their running power-law index model). Our results are thus also consistent with these $W M A P$ values at the less than $1.5 \sigma$ level.

\subsection{Temporal Stability of the Shear Measurement}

The CTE effect described in $\S 3.2$ is growing worse as the STIS CCD ages (Goudfrooij \& Kimble 2002). In order to test whether this degradation affects our results, we perform two tests. The first test involves stars, and the second test involves examining our final result for temporal stability.

There are sufficient stars in the survey to perform the analysis described in $\S 3.2$ on two subsections of the data. We divide the data roughly in half by time, classifying data taken before mid-1999 as "early" and data taken after that time as "late." We find that for $\mathrm{S} / \mathrm{N}>20$, there is little change to the fit to $e_{1}$ shown in Figure 3. However, for $\mathrm{S} / \mathrm{N}<20$, we find that the fits shown in Figure 3 do change slightly. The early data are fitted by curves with $e_{1}$ slightly higher than those 


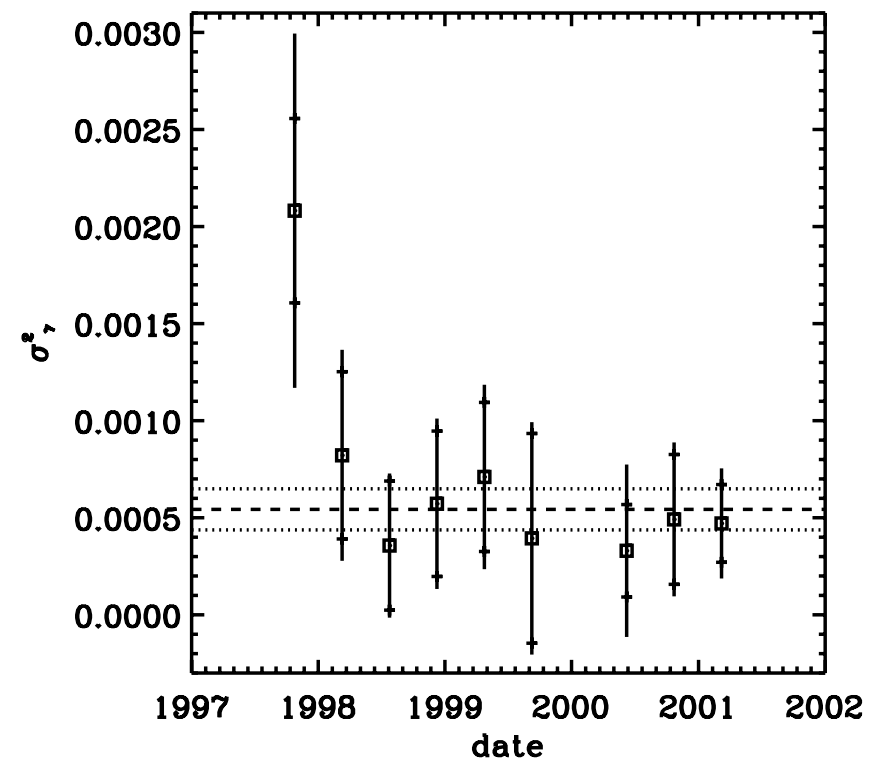

FIG. 5.-Cosmic shear variance $\sigma_{\text {lens }}^{2}$ as a function of the date. The inner error bars include statistical errors only, while the outer error bars include statistical errors and cosmic variance. The dotted horizontal lines represent the value of $\sigma_{\text {lens }}^{2}$ and the associated $1 \sigma$ statistical error for the entire survey. The leftmost point is consistent with what Hämmerle et al. (2002) found using roughly the same data.

shown in Figure 3, and the late data show a slightly lower $e_{1}$. This is consistent with a CTE that is growing worse over time. In both cases, the departure from the curve shown in Figure 3 is about $1 \%$. This average $1 \%$ systematic error in the correction of stellar ellipticities at low $\mathrm{S} / \mathrm{N}$ is included in the error estimates for our values of $\sigma_{\gamma}^{2}$ and $\sigma_{8}$ (see $\left.\S 4.1\right)$.

To perform the other test, we divide our galaxy data into nine equally spaced date bins. We then measure the rms shear $\sigma_{\gamma}^{2}$ in each bin. We show these measurements in Figure 5. This plot shows no trend over time, as might be expected if the CTE effect were not being sufficiently corrected for. The shear signal is consistent throughout the time in which the data we use in this paper were taken. The only marginal outlier is the very first bin, which is $1.7 \sigma$ from the value for the entire survey when statistical errors and cosmic variance are included. We have run several tests to determine why data taken during this period give a higher shear signal than data taken during the rest of the survey, but we find nothing special about the data in this bin. Our results in this date range are consistent with those of Hämmerle et al. (2002), who found a high shear signal using STIS data from roughly the same period. Excluding the first bin (by only analyzing data taken after 1997 December) only changes the measured $\sigma_{\gamma}^{2}$ from $(5.43 \pm 1.74) \times 10^{-4}$ to $(4.76 \pm 1.68) \times 10^{-4}$. The effect on $\sigma_{8}$ is less pronounced; the removal of the first bin decreases $\sigma_{8}$ from $1.02 \pm 0.16$ to $0.97 \pm 0.16$.

\section{CONCLUSIONS}

We have measured the shapes of 26,036 galaxies in 1292 STIS fields, corresponding to about $0.25 \mathrm{deg}^{2}$. We corrected for systematic effects, as outlined in RRG00, with the added step of correcting galaxy moments for an $\mathrm{S} / \mathrm{N}$-dependent charge transfer efficiency. We have detected a cosmic shear signal, using the STIS parallel archive at the $5.1 \sigma$ level. After correcting the galaxy shapes for PSF distortions, detector shear, and CTE effects, we measure a shear variance of $\sigma_{\text {lens }}=$ $(5.43 \pm 1.74) \times 10^{-4}$ on the STIS chip scale $\left(50^{\prime \prime}\right.$ on a side), where the $1 \sigma$ error includes noise, cosmic variance, and systematics. This is consistent with the earlier and much noisier measurement of Hämmerle et al. (2002). For a $\Lambda$ CDM model with $\Omega_{m}=0.3$ and $\Gamma=0.21$, this sets a constraint on the amplitude of the matter power spectrum given by $\sigma_{8}=1.02 \pm$ 0.16 , where the $1 \sigma$ error includes noise, cosmic variance, systematics, and redshift error. This is consistent with earlier measurements of $\sigma_{8}$ from cosmic shear but tends to favor those with higher values. It is also consistent with the recent determination of $\sigma_{8}$ (Spergel et al. 2003) from CMB anisotropies with the WMAP mission.

The results presented here represent a contribution to the first generation of space-based weak-lensing results presented in RRG01, RRG02, and Hämmerle et al. (2002). The next generation of space-based parallel observations optimized for weak lensing will be made with the Advanced Camera for Surveys (ACS) on the HST. A targeted ACS project, the COSMOS $2 \mathrm{deg}^{2}$ field (GO-9822) will provide strong constraints on cosmological parameters and allow for highresolution dark matter map. ACS surveys will benefit from the high resolution of ACS (0".05 pixels, like STIS), as well as its enlarged area (approximately $10 \mathrm{arcmin}^{2}$ ) and improved sensitivity (relative to WFPC2). New methods for the measurement of object shapes and the correction of systematic effects are being developed that will capitalize on the excellent resolution of this survey and future space-based surveys (Refregier \& Bacon 2003; Bernstein \& Jarvis 2002). Future generations of weak-lensing surveys, both from the ground (e.g., the CanadaFrance-Hawaii Legacy survey: Mellier et al. 2000; the Largeaperture Synoptic Survey Telescope) ${ }^{10}$ and from space (e.g., the Supernova/Acceleration Probe [SNAP]; C. Alcock et al. 2004, in preparation; Rhodes et al. 2004; Massey et al. 2003; Refregier et al. 2003), will continue to utilize this unique method to measure cosmological parameters to unprecedented accuracy. These projects will survey large areas while obtaining images in multiple filters, allowing for accurate photometric redshifts of the survey galaxies. SNAP, with its near-infrared capability, high resolution, and wide field of view, will be able to probe mass concentrations beyond $z=1$, thus allowing for redshift tomography and the study of the growth of structure. This will enable weak lensing to make a contribution to the study of dark matter and dark energy and to set tight constraints on cosmological parameters.

We thank Richard Massey and Richard Ellis for useful discussions. We thank the referee, Peter Schneider, for useful comments and suggestions. A. R. was supported in Cambridge by an EEC fellowship from the TMR network on Gravitational Lensing and by a Wolfson College Research Fellowship. E. J. G. was supported by NASA grant NAG5-6279. J. R. was supported by an National Research Council-GSFC Research Associateship. We thank Bruce Woodgate, Elliot Malumuth, Randy Kimble, Ted Gull, Terry Beck, Keith Feggans, and the rest of the STIS team at GSFC for their support in cataloging and understanding the STIS parallel images.

\footnotetext{
${ }^{10}$ For the Canada-France-Hawaii Telescope Legacy survey, see http:// cdsweb.u-strasbg.fr:2001/Science/CFHLS. For the the Large-Aperture Synoptic Survey Telescope, see http://www.lssto.org/Science/darkmatter.html.
} 


\section{REFERENCES}

Bacon, D. J., Massey, R. J., Refregier, A. R., \& Ellis, R. S. 2003, MNRAS, 344,673

Bacon, D. J., Refregier, A. R., \& Ellis, R. S. 2000, MNRAS, 318, 625

Baum, S. A., et al. 1996, STIS Instrument Handbook, Version 1.0 (Baltimore: STScI)

Bernstein, G. M., \& Jarvis, M. 2002, AJ, 123, 583

Bertin, E., \& Arnouts, S. 1996, A\&AS, 117, 393

Brown, M. L., Taylor, A. N., Bacon, D. J., Gray, M. E., Dye, S., Meisenheimer, K., \& Wolf, C. 2003, MNRAS, 341, 100

Brown, T. 2001, STIS Instrum. Sci. Rep. 2001-3 (Baltimore: STScI)

Gardner, J. P., et al. 1998, ApJ, 492, L99

Goudfrooij, P., \& Kimble, R. 2002, in The 2002 HST Calibration Workshop, ed. S. Arribas, A. Koekemoer, \& B. Whitmore (Baltimore: STScI), 105

Hamana, T., et al. 2003, ApJ, 597, 98

Hämmerle, T., et al. 2002, A\&A, 385, 743

Hoekstra, H., Yee, H. K. C., Gladders, M. D., Barrientos, L. F., Hall, P. B., \& Infante, L. 2002, ApJ, 572, 55

Jarvis, M., Bernstein, G. M., Fischer, P., Smith, D., Jain, B., Tyson, J. A., \& Wittman, D. 2003, AJ, 125, 1014

Kimble, R., Goudfrooij, P., \& Gilliland, R. L. 2000, Proc. SPIE, 4013, 532

Kron, R. G. 1980, ApJS, 43, 305

Lindler, D. 2003, CALSTIS Reference Guide (Greenbelt: GSFC)

Malumuth, E. M., \& Bowers, C. W. 1997, in The 1997 HST Calibration Workshop, ed. S. Casertano, R. Jedrzejewski, C. D. Keyes, \& M. Stevens (Baltimore: STScI), 144
Massey, R., et al. 2003, AJ, submitted (astro-ph/0304418)

Mellier, Y., van Waerbeke, L., Bernardeau, F., \& Tenero, I. 2003, in Proc. ESO-CERN-ESA Symp., Astronomy, Cosmology, and Fundamental Physics, ed. P. A. Shaver, L. Di Lella, \& A. Giménez (Berlin: Springer), 25

Mellier, Y., et al. 2000, in Mining the Sky, ed. A. J. Banday, S. Zaroubi, \& M. Bartelmann (Heidelberg: Springer), 540

Nelan, E., \& Makidon, R. B. 2002, in HST Data Handbook for FGS, Version 4.0, ed. B. Mobasher (Baltimore: STScI)

Pirzkal, N., et al. 2001, A\&A, 375, 351

Refregier, A. 2003, MNRAS, 338, 35

Refregier, A., \& Bacon, D. 2003, MNRAS, 338, 48

Refregier, A., Rhodes, J., \& Groth, E. J. 2002, ApJ, 572, L131 (RRG02)

Refregier, A., et al. 2003, AJ, submitted (astro-ph/0304419)

Rhodes, J. Refregier, A., \& Groth, E. J. 2000, ApJ, 536, 79 (RRG00) 2001, ApJ, 552, L85 (RRG01)

Rhodes, J., et al. 2004, Astropart. Phys., 20, 337

Smith, R. E., et al. 2003, MNRAS, 341, 1311

Spergel, D., et al. 2003, ApJS, 148, 175

Stetson, P. B. 1987, PASP, 99, 191

Teplitz, H. I., Collins, N. R., Gardner, J. P., Hill, R. S., Heap, S. R., Lindler, D. J., Rhodes, J. D., \& Woodgate, B. E. 2003a, ApJS, 146, 209 Teplitz, H. I., Collins, N. R., Gardner, J. P., Hill, R. S., \& Rhodes, J. 2003b, ApJ, 589, 704

van Waerbeke, L., Mellier, Y., Pelló, R., Pen, U.-L., McCracken, H. J., \& Jain, B. 2002, A\&A, 393, 369 